\title{
Topology optimization for simplified structural fire safety
}

Madsen, Søren; Lange, Nis P.; Giuliani, Luisa; Jomaas, Grunde; Lazarov, Boyan Stefanov; Sigmund, Ole

\section{Published in:}

Engineering Structures

Link to article, DOI:

10.1016/j.engstruct.2016.06.018

Publication date:

2016

Document Version

Peer reviewed version

Link back to DTU Orbit

Citation (APA):

Madsen, S., Lange, N. P., Giuliani, L., Jomaas, G., Lazarov, B. S., \& Sigmund, O. (2016). Topology optimization for simplified structural fire safety. Engineering Structures, 124, 333-343.

https://doi.org/10.1016/j.engstruct.2016.06.018

\section{General rights}

Copyright and moral rights for the publications made accessible in the public portal are retained by the authors and/or other copyright owners and it is a condition of accessing publications that users recognise and abide by the legal requirements associated with these rights.

- Users may download and print one copy of any publication from the public portal for the purpose of private study or research.

- You may not further distribute the material or use it for any profit-making activity or commercial gain

- You may freely distribute the URL identifying the publication in the public portal 


\title{
Topology Optimization for Simplified Structural Fire Safety
}

\author{
Søren Madsen ${ }^{\mathrm{a}}$, Nis P. Lange ${ }^{\mathrm{a}}$, Luisa Giuliani ${ }^{\mathrm{b}}$, Grunde Jomaas ${ }^{\mathrm{b}}$, Boyan S. Lazarov ${ }^{\mathrm{a}}$, Ole Sigmund ${ }^{\mathrm{a}}$ \\ ${ }^{a}$ Department of Mechanical Engineering, Solid Mechanics, \\ Technical University of Denmark, Niels Koppels Alle, B.404, \\ DK-2800 Lyngby, Denmark \\ ${ }^{b}$ Department of Civil Engineering, \\ Technical University of Denmark, Brovej, B. 118 \\ DK-2800 Lyngby, Denmark
}

\begin{abstract}
Topology optimization is applied in an idealized structural fire safety model, where the minimum compliance problem is constrained by temperature-controlled structural degradation. The constraint ensures a certain structural stiffness after a prescribed time. As this time period is extended, resulting optimized topologies tend to become thicker or introduce redundant members that can take over when structural parts near the origin of the fire lose their load carrying capability. Hence, the structural degradation model acts as an erosion operator on the topology and indirectly enforces a minimum length scale on the final designs.
\end{abstract}

Keywords: Topology optimization, Multiphysics, Structural fire safety, Length scale, Robustness

\section{Introduction}

\subsection{Object and motivation}

Structural design aims at providing sufficient load carrying capacity for the Ultimate Limit State (ULS) and appropriate stiffness for the Serviceability Limit State (SLS). In addition, structural integrity against accidental events, such as fire, explosions, and unexpected local failures, must be ensured. This third requirement is included in the Eurocodes [1] as an accidental design situation and commonly referred to as the Accidental Limit State (ALS). It is generally difficult to account for all three limit state requirements in the early stages of structural design. Therefore, optimizations associated with the ULS and SLS are typically performed first, and later, if necessary, the design is adapted and verified for accidental actions. Such amendments in the late stages of the design process may affect the optimality of the already optimized design. This is especially true for the case of fire design, because the design characteristics of fire resistant elements often are opposite to those of elements optimized for stiffness and resistance at the SLS and ULS.

In order to clarify the latter statement, a typical design optimization problem can be considered, where, given a certain amount of steel, the optimal shape of a section profile is sought. Table 1 provides an overview of profiles with the most common geometrical shapes and the same area (small deviations in the values are given by limitations on the dimensions of existing profiles). For the sake of simplicity, only sections having the same maximum dimensions $\left(L_{x}, L_{y}\right)$ along the two principal axes are considered and analyzed with respect to the resistance to a positive bending moment. The HEB (European wide flange H-beams) profile has by far the highest elastic modulus of resistance $W_{e l}$, showing that the best way of distributing the steel for maximizing the resistance at ambient condition is to concentrate it at the top and bottom of the domain, where the elastic stresses are the highest. On the other hand, concentrating the steel closer to the core of the profile, such as in a triangular- or diamond-shaped profile, yields a high plastic benefit, intended as ratio between the plastic and elastic modulus of resistance $W_{p l} / W_{e l}$. However, the shape that yields the highest plastic modulus of resistance is still the HEB profile, which is therefore optimal from the point of view of

Email address: sigmund@mek.dtu.dk (Ole Sigmund) 


\begin{tabular}{|c|c|c|c|c|c|c|c|c|c|}
\hline $\begin{array}{l}\text { Profile } \\
\text { shape }\end{array}$ & $\begin{array}{c}L_{y}=L_{x} \\
{[\mathrm{~m}]}\end{array}$ & $\begin{array}{l}\text { thickness } \\
{[\mathrm{m}]}\end{array}$ & $\begin{array}{c}\mathrm{A} \\
{\left[\mathrm{m}^{2}\right]}\end{array}$ & $\begin{array}{c}W_{e l} \\
{\left[\mathrm{~m}^{3}\right]}\end{array}$ & $\begin{array}{l}W_{p l} \\
{\left[\mathrm{~m}^{3}\right]}\end{array}$ & $\begin{array}{c}W_{p l} / W_{e l} \\
{[-]}\end{array}$ & $\begin{array}{c}P / A \\
{\left[\mathrm{~m}^{-1}\right]}\end{array}$ & $\begin{array}{c}f_{y}\left(t=30^{\prime}\right) / f_{y} \\
{[-]}\end{array}$ & $\begin{array}{c}M_{p l}\left(t=30^{\prime}\right) / f_{y} \\
{\left[\mathrm{~m}^{3}\right]}\end{array}$ \\
\hline CIRCULAR & $1.35 \mathrm{E}-01$ & - & $1.43 \mathrm{E}-02$ & $2.41 \mathrm{E}-04$ & $4.09 \mathrm{E}-04$ & 1.7 & 29.7 & $50 \%$ & $2.06 \mathrm{E}-04$ \\
\hline ISOSC. TRIAN. & $1.70 \mathrm{E}-01$ & - & $1.45 \mathrm{E}-02$ & $4.09 \mathrm{E}-04$ & $9.70 \mathrm{E}-04$ & 2.4 & 38.1 & $21 \%$ & 2.03E-04 \\
\hline SQUARED & $1.20 \mathrm{E}-01$ & - & $1.44 \mathrm{E}-02$ & $2.88 \mathrm{E}-04$ & 4.32E-04 & 1.5 & 33.3 & $34 \%$ & $1.46 \mathrm{E}-04$ \\
\hline HEB300 & $3.00 \mathrm{E}-01$ & $0.11 \mathrm{E}-01$ & $1.43 \mathrm{E}-02$ & $1.61 \mathrm{E}-03$ & $1.79 \mathrm{E}-03$ & 1.1 & 123.4 & $7 \%$ & $1.20 \mathrm{E}-04$ \\
\hline HOLLOW CIRC. & $2.13 \mathrm{E}-01$ & $2.50 \mathrm{E}-02$ & $1.48 \mathrm{E}-02$ & $6.23 \mathrm{E}-04$ & 7.92E-04 & 1.3 & 45.3 & $13 \%$ & $1.06 \mathrm{E}-04$ \\
\hline HOLLOW RECT. & $2.00 \mathrm{E}-01$ & $2.00 \mathrm{E}-02$ & $1.44 \mathrm{E}-02$ & 7.87E-04 & $9.76 \mathrm{E}-04$ & 1.2 & 55.6 & $9 \%$ & $9.16 \mathrm{E}-05$ \\
\hline
\end{tabular}

Table 1: Elastic, plastic, and fire resistance properties for profiles with different shapes. $f_{y}$ denotes yield stress and $M_{p l}$ denotes sectional plastic moment.

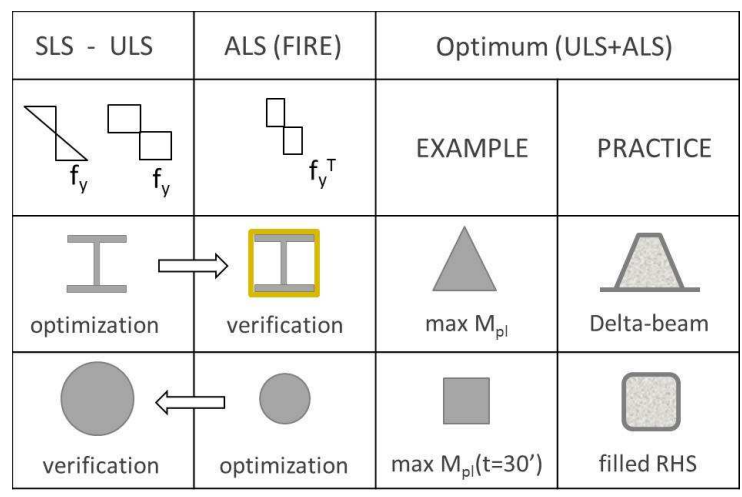

Figure 1: Optimization of steel beam profiles at the different limit states.

both SLS and ULS. This is not the case anymore when fire is considered, because the concentration of the steel area towards the core of the profile has a double effect, as it also reduces the ratio between the exposed perimeter and the area of the profile $(P / A)$, referred in the following as the section factor. Hereby, the temperature of the profile for a given time of fire exposure is also reduced and the degradation of the steel's mechanical properties is less severe. If a standard fire exposure of 30 minutes is considered, a circular shape is the optimal profile, closely followed by the triangular-shaped profile. On the other hand, the HEB profile has by far the highest section factor and the most severe reduction of strength $f_{y}\left(t=30^{\prime}\right) / f_{y}$, as well as one of the lowest fire resistances (followed only by the hollow circular and rectangular profiles). In the example, the strength reduction for the $2 \%$ stress, i.e. effective yielding, is calculated with the formula suggested by Hertz [2].

As a result, an optimization based on SLS or ULS designs would require a significant amount of insulation in order to provide the HEB profile with sufficient resistance in case of a fire (right arrow on second row of Figure 1). Therefore, steel profiles are typically insulated by fire-resistant panels, intumescent paints or spray-on plasters. The first method deceives the aesthetic aspects of the optimized structures, the second nullifies the original economic benefits, and the third is non-ideal from both an aesthetic and an economic perspective. Conversely, an optimization based exclusively on the fire resistance would hardly comply with the stiffness or ULS resistance requirements and ultimately lead to a cumbersome increment of the cross-section dimensions and an uneconomical design of the profiles. This is exemplified by the left arrow on the third row of Figure 1, where a circular profile is chosen from an optimization based only the fire resistance and then the dimension of the profile is increased for satisfying the ULS verification. The optimal profile can only be identified by performing the optimization at a later design stage, i.e. after both stiffness and fire resistance requirements have been verified. By assuming a maximum strength reduction factor of $30 \%$ (on the basis of a typical reduction factor of the load for the fire case [3]), a later optimization of either the resistance (max $M_{p l}$ ) or the fire resistance $\left(\max M_{p l}\left(t=30^{\prime}\right)\right.$ ) would lead to a selection of the rectangular and triangular profile as optimal, respectively. Even if only simple, homogeneous, and not necessarily realistic geometrical shapes have been considered in this example, the triangular and rectangular profiles relate very well with common profiles for structural fire safety, trapezoidal or rectangular (RHS) hollow section filled with light concrete.

This simple example for the identification of an optimum steel profile points out the need for developing a unified structural design approach that takes all design requirements into account already in the initial design stage. The main objective of this work is to present a systematic way of taking fire safety related considerations into account in 
the early stages of structural design. The authors acknowledge that this goal is a huge, complicated and challenging endeavor and hence the present work shall only be seen as a first step in this direction.

\subsection{Background and method}

The approach is based on topology optimization, a systematic tool for structural and mechanical design initiated by Bendsøe and Kikuchi [4] and since then extended to all kinds of other applications [5]. The approach is based on an iterative procedure including finite element analyses, gradient computations and design updates by Mathematical Programming approaches, where the design variables represent relative material density in each finite element used to discretize the design domain. In this way, one can obtain optimized structures represented by bit or voxel-maps without any restrictions on geometrical freedom. If needed, one may impose various kinds of length-scale control or robustness to manufacturing variations [6, 7]. The literature on topology optimization is abundant and here we only list a few references of relevance to the present work. Topology optimization for thermal loading was initiated by Rodrigues and Fernandes [8] and extended to material design by Sigmund and Torquato [9] and to MEMS structures [10]. Convection effects have been included first in topology optimization of micromechanisms [11], and later in the design of general multiphysical systems [12, 13, 14]. Topology optimization for transient problems was developed for dielectric problems [15, 16, 17], thermal problems [18] and crashworthiness problems [19].

The topic of topology optimization for structural fire safety has seemingly not been the subject of much research, but a simple approach is suggested by Diaz and Benard [20]. Based on an uncoupled static mechanical problem and a steady-state heat transfer problem, the standard minimum compliance problem is extended with a maximum temperature constraint. Operating with two materials, a structural material and a thermally insulating material, it is shown how the latter will encase the former in order to protect it from heat.

Herein, another method is presented based on a weakly coupled static mechanical problem and transient heat transfer problem, in which the pursuit of maximum stiffness is constrained by a time-dependent structural degradation. The degradation starts from the surfaces exposed to fire and eats more and more of the structure as time passes and the material points reach a certain temperature. This simplified, and so far rather academic, formulation is used to obtain topologies that are capable of resisting heat controlled degradation for a given duration of time.

\subsection{Structure of the paper}

Section 2 introduces the design approaches used in structural fire safety and presents the simplified fire and material model used in the study. Section 3 gives details on the topology optimization formulation, finite element analysis and sensitivity analysis as well as on regularization schemes for ensuring stable topology optimization solutions. Section 4 demonstrates the approach by two examples for design of structural components engulfed in fire and one structure subjected to a localized fire. Section 5 concludes on approach and results and discusses necessary steps for the extension of the present purely academic state of research to a more practically oriented and applicable approach.

\section{Structural fire design}

\subsection{Post-flashover design}

The behavior of a fire in an enclosed building environment is very complex and depends on several factors: the properties of the fuels (type and amount) and compartments (geometry, linings, and ventilation) affect the peak temperature and the duration of the fire; the presence and activation of fire extinguishment systems, as well as the fire brigade's intervention, reduce the probability that a local fire grows to involve the entire compartment of origin. The radiation from the hot smoke layer causes the ignition of all combustible surfaces. This phenomenon is referred to as flashover and the fire is said to be fully developed. At this point, a unique temperature can be assumed for the whole compartment and used for assessing the heating of the structural elements (Figure 2). The temperature-time curve of the compartment is calculated by equating the heat produced per unit time to the heat dissipated through radiation, convection and conduction through the openings and the walls [21]. Having a heating phase, a peak, and a cooling phase determined by the above mentioned fuel and compartment characteristics, these curves are referred to as parametric fires, e.g., see Figure 2.

An alternative, simpler post-flashover fire model has been developed by assuming a monotonically increasing curve, which envelopes all parametric curves obtained for the most common compartments. This curve, referred 


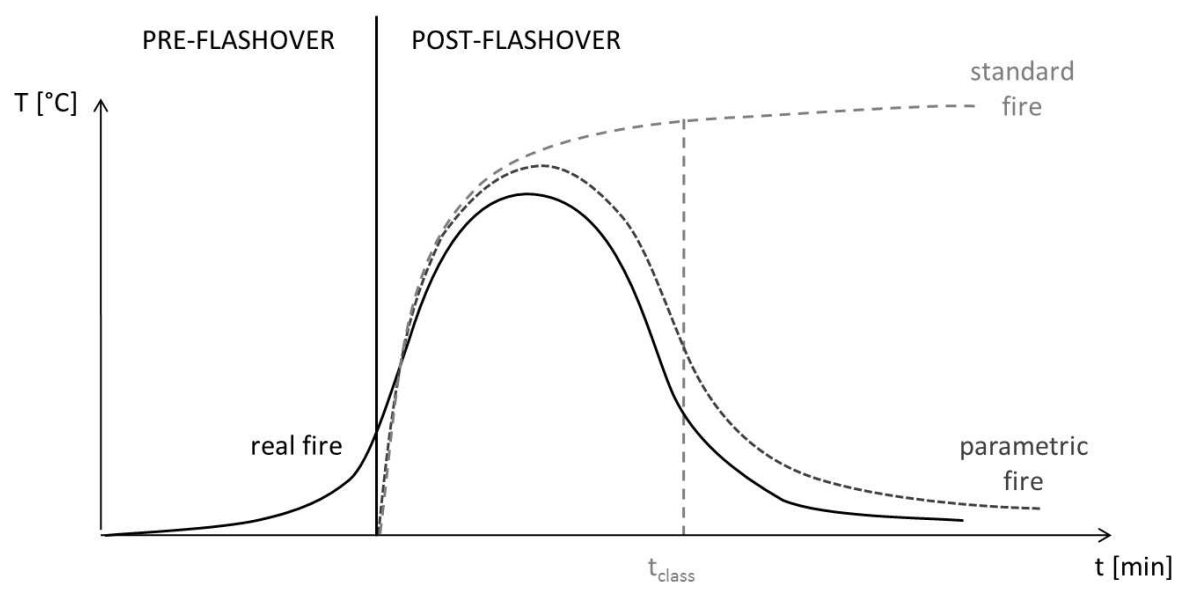

Figure 2: Time-temperature curves for a real fire and post-flashover design fires (e.g. [28]).

to as the ISO834 curve or the standard fire curve [22], is clearly less realistic than a parametric fire, but is often used in the design practice in virtue of its independency from the building where the structural elements will be placed. This allows for a direct comparison of different design solutions and for the definition of simple prescriptive requirements, based on the resistance time of the structural elements to this nominal fire. Generally, intervals of 15 min are considered, so that the following structural classes $\mathrm{R} 15, \mathrm{R} 30, \ldots, \mathrm{R} 120$ are assigned to elements maintaining their load-bearing capacity, respectively, at least $15,30, \ldots$ and 120 minutes when exposed to a standard fire in a test furnace. The requirements for resistance classes of the elements are defined at national level on the basis of the building type and compartment occupancy [23, 24].

When the resistance class design approach is followed, the Eurocodes [23] allow neglecting the indirect stresses induced by the part of thermal expansion that is hindered by adjoining elements. This is permitted in virtue of the supposed conservativeness of the standard fire and determines a significant simplification of the design, which can be carried out on single structural elements. Nevertheless, hindered thermal expansion is known to strongly influence the fire resistance and the collapse mode of structures [25] and to be one of the most significant factors causing fire-induced collapses of buildings [26]. It should also be noted that the effective conservativeness of the resistance class method depends entirely on the establishment of appropriate resistance classes [27], as the energy content of the standard fire is determined entirely by the prescribed resistance time.

\subsection{Localized fires and structural integrity}

A further limitation of structural fire design based either on standard or on parametric fires lies in the implicit assumption of flashover, which rarely occurs in large or well-ventilated compartments, i.e., in compartments where there is always a sufficient amount of oxidizer for complete combustion of the burning materials. This is the case for open-office compartments or atria, which are often used in modern buildings, as well as industrial halls and open or semi-open car parks. Despite the fact that the assumption of flashover in most cases leads to a conservative design [29], some recent studies have highlighted how the method may lead to a strong underestimation of the element temperatures for compartments where a traveling fire develops [30]. Current building codes limit the applicability of standard and parametric fires to compartments with a floor area smaller than $500 \mathrm{~m}^{2}$ [23, 24] , but do not give guidance on how to perform a safe design of larger compartments. For this reason, the consideration of pre-flashover localized fire models for structural design is of increasing interest.

It is noted that, in case of a localized fire, the failure of few elements directly exposed to the fire may be accepted, as long as it does not affect the global stability of the structure. This requirement, often referred to as structural integrity or robustness, is demanded by most international building codes [31, 32, 33] and is of particular importance when designing for accidental actions such as fires. This is because the risk of limited economic consequence can be accepted for events that have a low probability of occurrence. 


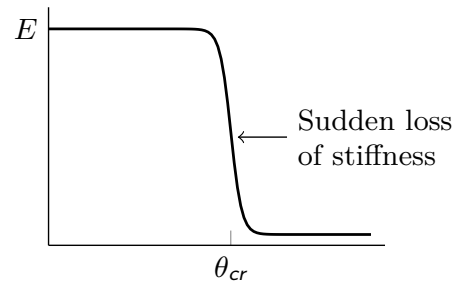

Figure 3: Fire degradation model.

However, since the response of the whole or a large part of the structure must be investigated, this kind of design is much more complex than the usual fire design, aimed at avoiding the failure of each single structural component either for a given time of exposure to the standard fire (resistance class method) or for the whole duration of a compartment fire (parametric fire method). As such, this advanced design is hardly performed in the usual practice and mostly limited to complex or special structures.

The method presented herein aims to be applicable to the case of both local and structural fires and to integrate in the same general procedure the evaluation of the fire resistance of individual components and of the structural integrity of larger structures.

\subsection{An idealized fire and material model}

Herein, a fire is modeled as a transient heat transfer problem weakly coupled to a static mechanical problem. A convection controlled heat flow, $q$, is generated across the structural surfaces, representing the occupation of hot gases in all available space:

$$
q=h_{0}\left(\theta_{\infty}-\theta_{0}\right)
$$

where $h_{0}$ is a convection coefficient governing the heat transfer rate, $\theta_{\infty}$ is the temperature of the surrounding gases and $\theta_{0}$ is the surface temperature of the structure. An arbitrary, isotropic material is considered and the impact of the fire is simplified as explained in the following. As illustrated in Figure 3, a critical temperature $\theta_{c r}$ is introduced, at which the material looses (almost) all stiffness. Hence, the applied convection causes a time-dependent structural degradation. Also, thermal expansion is ignored herein and thermal conductivity and specific heat capacity are assumed constant. However, it would be rather simple to include more advanced models in the non-linear, time-dependent formulation considered.

As illustrated in Figure 4, this model may approximate a fully exposed truss beam (as seen in industrial storage buildings) made of an isotropic material such as steel in a post-flashover fire situation. In this case, convection is applied across the surfaces of the statically loaded structure (Figure 4 a), representing the occupation of hot gases in the whole domain. The rising temperatures cause a time-dependent structural degradation, which is illustrated by the gray area in Figure 4 b) and Figure 4 c) for increasing duration of the fire. Local fires may also be represented by simply applying convection across the surfaces of a limited area of the domain, corresponding to the location of the fire. It should be noted that, despite the fact that the example presented in the following assumes a constant temperature of the heating source, a more realistic fire model can be obtained by including a standard, parametric or local fire as a time dependent temperature function.

\subsection{Objective function}

As the focus here is on both robustness of larger structures and heat resistance of individual structural components, a modified stiffness-based approach is utilized. A usual problem in structural topology optimization is to minimize compliance subject to a volume constraint. In the present case, this corresponds to maximizing the stiffness of the undamaged structure for a given amount of material. In addition, we require the structural stiffness, after a certain time of fire exposure, to be at least a factor $R_{s}$ times the original stiffness. A fire safety factor of $R_{s}=1$ means that no structural degradation is allowed, whereas $R_{s}=0$ means that the structure is allowed to loose all stiffness. 


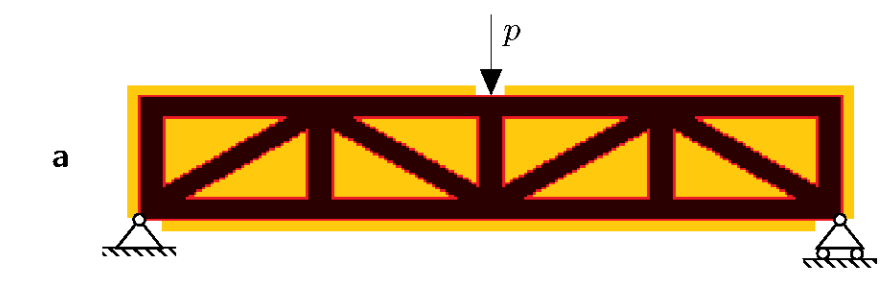

$\mathbf{b}$

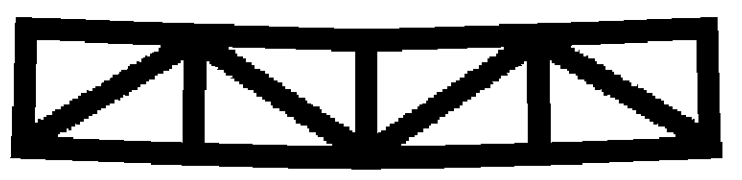

C

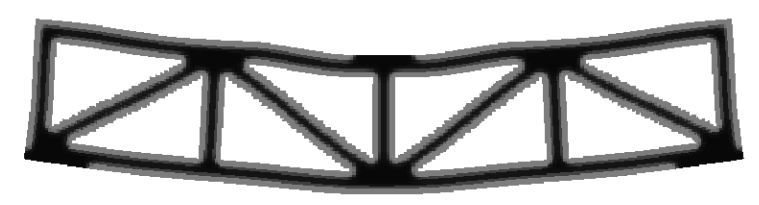

Figure 4: Convection is applied across the surfaces of the statically loaded structure a, representing the occupation of hot gases in all available space. The rising temperatures cause time-dependent structural degradation, b-c, where gray-scale illustrates degraded material after a certain duration of fire.

The constrained minimum compliance problem is defined as:

$$
\begin{aligned}
& \min .: \Phi_{0} \\
& \text { s.t. : } \Phi_{j} \leq \frac{\Phi_{0}}{R_{S}}, j=1, \ldots, M
\end{aligned}
$$

where $\Phi_{0}$ and $\Phi_{j}$ are the compliances before and after the impact of $M$ fire scenarios, respectively. Here $M$ is either a number of local fire scenarios, which is relevant for assessing the robustness of a larger structure, or for $M=1$, a post-flashover fire, which is relevant for assessing the heat resistance of a structural component (as exemplified in Figure 4). The optimization problem above can be reformulated to the so-called bound formulation as:

$$
\begin{aligned}
\min . & : b \\
\text { s.t. } & : \Phi_{0} \leq b \\
& : R_{s} \cdot \Phi_{j} \leq b, j=1, \ldots, M
\end{aligned}
$$

It should be noted that Starossek and Haberland [34] stress the fact that there is a relatively bad correlation between the stiffness-based measure and the remaining load carrying capacity, which is the true measure for structural integrity. Nevertheless, we use it here due to relatively easy integration in a topology optimization algorithm, recognizing that this is a first step towards a more comprehensive approach.

\section{Topology optimization approach}

We consider two-dimensional problems where the design domain $\Omega$ subjected to thermal and mechanical boundary conditions is shown in Figure 5. The domain is occupied by a solid phase, $\Omega_{0}$, and a void phase, $\Omega_{\text {air }}$. The solid phase is bounded by the external and internal, physical boundaries $\Gamma_{0, \text { ext }}$ and $\Gamma_{0, \text { int }}$, respectively. Convection is applied across the internal and external surfaces of the solid phase. In the first examples convection is also applied on the lateral surfaces (top and bottom surfaces) simulating structural elements of finite depth, engulfed in fire. Convection is not applied to the lateral surfaces in the last example, simulating a deep building structure only subjected to internal fire. A time dependent structural degradation is modeled by a weakly coupled static mechanical and transient heat-transfer 
problem. The aim is to optimize the distribution of a limited amount of material, e.g. the layout of $\Omega_{0}$, such that the maximum between the undamaged compliance at $t=t_{0}$ and the hot compliance at time $t^{*}$ multiplied by the fire safety factor $R_{s}$ is minimized (c.f. Equation 2). The time $t^{*}$ can be interpreted from a design point of view as the resistance class required for a structural component, which has been discussed in the introduction.

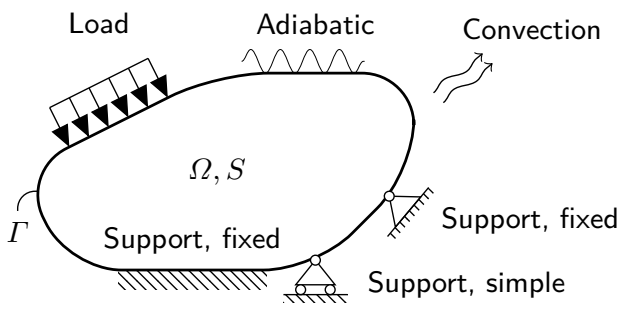

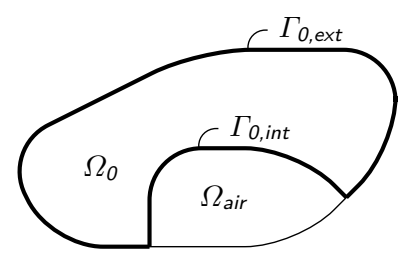

b

Figure 5: a: The design domain $\Omega$ is bounded by $\Gamma$ and has the lateral surfaces $S . \Omega$ is subjected to mechanical and thermal, boundary conditions. b: Subdivision of the design domain.

\subsection{Mathematical formulation}

The system response is governed by the following set of differential equations [35]:

$$
\left\{\begin{array}{l}
\mathbf{C}(\rho) \dot{\theta}+\mathbf{K}_{\theta}(\rho) \boldsymbol{\theta}=\mathbf{p}_{\theta}(\rho) \\
\mathbf{K}(\rho, \boldsymbol{\theta}) \mathbf{u}=\mathbf{p}
\end{array}\right.
$$

where: $\boldsymbol{\theta}$ and $\mathbf{u}$ are the temperature field and displacement field vectors, respectively; $\mathbf{p}_{\theta}$ and $\mathbf{p}$ are the thermal and mechanical load vectors; $\mathbf{C}, \mathbf{K}_{\theta}$ and $\mathbf{K}$ are the thermal capacity, heat conduction and stiffness matrices, respectively. The material distribution is described by the design variables $\boldsymbol{\rho}$. To each element $i$ a design variable is assigned $\rho_{i} \in[0,1]$, which governs its thermal conductivity and specific heat through the interpolations:

$$
\begin{aligned}
& k_{i}\left(\rho_{i}\right)=k_{\text {min }}+\rho_{i}\left(k_{0}-k_{\min }\right) \\
& c_{i}\left(\rho_{i}\right)=c_{\text {min }}+\rho_{i}\left(c_{0}-c_{\text {min }}\right)
\end{aligned}
$$

where $k_{0}$ and $c_{0}$ are the material thermal conductivity and specific heat, respectively, and $k_{\min }$ and $c_{\min }$ are small values to prevent the conductivity and thermal capacity matrices from becoming singular. The Young's modulus is governed by the element design variable, following the modified SIMP approach [36], and the element temperature, $\theta_{i}$ :

$$
E_{i}\left(\rho_{i}, \theta_{i}\right)=E_{\min }+\rho_{i}^{p}\left(E_{0}-E_{\min }\right) \cdot H_{\theta}\left(\theta_{i}\right)
$$

where $E_{\min }$ is a small number to prevent the stiffness matrix from becoming singular and $H_{\theta}$ is a temperature dependent smoothed Heaviside step function, employing a sudden loss of stiffness, if the element temperature surpasses $\theta_{c r}$ (see Figure 3):

$$
H_{\theta}\left(\theta_{i}\right)=\frac{1}{1+\mathrm{e}^{\alpha\left(\theta_{i}-\theta_{c r}\right)}}
$$

The penalization factor, $p=3$, is introduced to steer the solution towards black and white designs and in all examples $\alpha=25 / \theta_{c r}$. The technique is similar to the one proposed for modeling the material degradation in topology optimization problems that consider stress induced damage [37], as well as in optimization under manufacturing uncertainties and length scale control [7].

The design variables also determine a convection controlled heat generation across the surface of the solid phase, $\Omega_{0}$ (see Figure 5). As illustrated in Figure 6, this includes the following three different boundary types:

1. Boundary Side Convection (BSC) across the external physical boundary, $\Gamma_{0, \text { ext }}$

2. Internal Side Convection (ISC) across the internal physical boundary, $\Gamma_{0, \text { int }}$

3. Top and Bottom Convection (TBC) across the two lateral surfaces of $\Omega_{0}$. 
TBC and BSC contribute to the element conductivity matrix and element thermal load vectors for element $i$, with the terms:

$$
\begin{aligned}
\mathbf{K}_{\theta, T B C, e i}\left(\rho_{i}\right) & =\int_{S_{i}} h_{i}\left(\rho_{i}\right) \mathbf{N}^{\top} \mathbf{N} \mathrm{d} S \\
\mathbf{p}_{\theta, T B C, e i}\left(\rho_{i}\right) & =\theta_{\infty} \int_{S_{i}} h_{i}\left(\rho_{i}\right) \mathbf{N}^{\top} \mathrm{d} S \\
\mathbf{K}_{\theta, B S C, e i}\left(\rho_{i}\right) & =\int_{\Gamma_{i}} h_{i}\left(\rho_{i}\right) d \mathbf{N}^{\top} \mathbf{N} \mathrm{d} \Gamma \\
\mathbf{p}_{\theta, B S C, e i}\left(\rho_{i}\right) & =\theta_{\infty} \int_{\Gamma_{i}} h_{i}\left(\rho_{i}\right) d \mathbf{N}^{\top} \mathrm{d} \Gamma
\end{aligned}
$$

where $\Gamma_{i}, S_{i}$ and $d$ are the boundary, lateral surfaces, and depth of the design domain for element $i$, respectively, $\theta_{\infty}$ is the temperature of the surrounding gases and $\mathbf{N}$ are the element shape functions. The convection coefficient is interpolated linearly as a function of the design variables:

$$
h_{i}\left(\rho_{i}\right)=h_{\min }+\rho_{i}\left(h_{0}-h_{\min }\right)
$$

where $h_{0}$ is the material convection coefficient and $h_{\min }$ is a small number, introduced to avoid numerical errors.

For ISC, the tricky part consists in finding the internal physical boundary, $\Gamma_{0 \text {,int }}$, as it appears and relocates during the optimization. Bruns [13] and Alexandersen [14] suggest a density-difference-based interpolation scheme, which is used here. A function $g_{I S C}$ is introduced:

$$
g_{I S C}(\Delta \rho)=\sqrt{(\Delta \rho)^{2}+\varepsilon^{2}}-\varepsilon \approx|\Delta \rho|
$$

where $\Delta \rho=\rho_{i}-\rho_{j}$ is the density difference between element $i$ and interface element $j$ and $\varepsilon$ is a small number, e.g. $10^{-3}$, introduced to make $g_{I S C}$ differentiable. The convection coefficient, $h$, is linearly interpolated between $h_{\min }$ and $h_{0}$ as a function of the density difference. The ISC contribution to the element conductivity matrix and element thermal load vector for element $i$ is derived as the combined contribution from all its interfaces:

$$
\begin{array}{r}
\mathbf{K}_{\theta, I S C, e i}\left(\boldsymbol{\rho}_{i}\right)=\frac{1}{2} \sum_{j=1}^{n_{\text {int }}} \int_{\Gamma_{i j}}\left(h_{\min }+g_{I S C}\left(\rho_{i}-\rho_{j}\right)\right. \\
\left.\left(h_{0}-h_{m i n}\right)\right) d \mathbf{N}^{\top} \mathbf{N} \mathrm{d} \Gamma_{i j} \\
\mathbf{p}_{\theta, I S C, e i}\left(\boldsymbol{\rho}_{i}\right)=\frac{1}{2} \sum_{j=1}^{n_{\text {int }}} \int_{\Gamma_{i j}}\left(h_{\min }+g_{I S C}\left(\rho_{i}-\rho_{j}\right)\right. \\
\left.\left(h_{0}-h_{\text {min }}\right)\right) d \mathbf{N}^{\top} \mathrm{d} \Gamma_{i j}
\end{array}
$$

where $n_{\text {int }}$ is the number of interfacing elements, $\boldsymbol{\rho}_{i}$ is the design variables of element $i$ and its interfacing elements and $\Gamma_{i j}$ is the internal boundary between element $i$ and $j$ (see Figure 6 $\mathrm{c}$ ). The factor $\frac{1}{2}$ is due to both interfacing elements $i$ and $j$ contributing to the convection across their joint boundary. Lumping of the heat capacity and boundary convection matrices is employed in order to alleviate numerical errors, as suggested by Bruns [13]. 


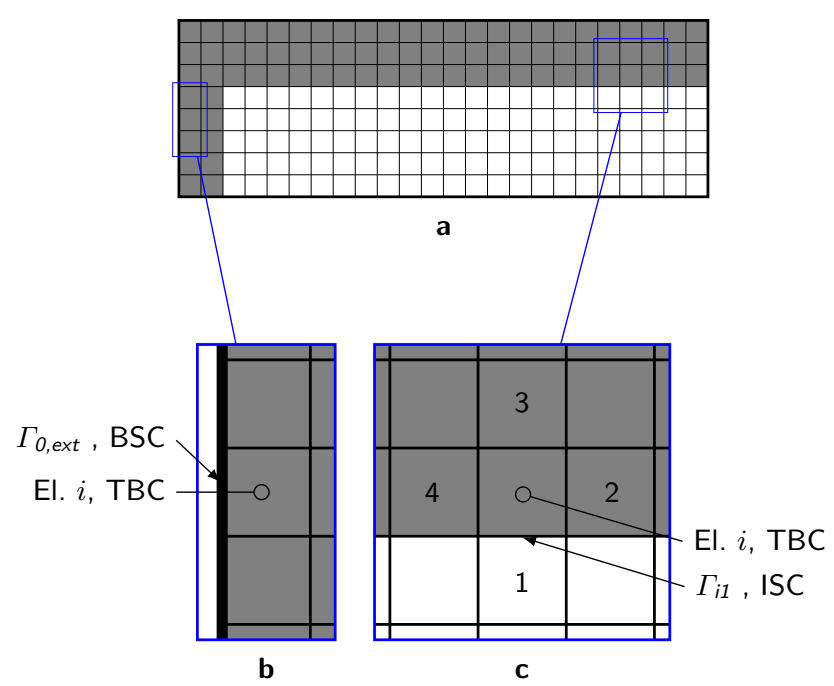

Figure 6: Convection is applied across the surface of the arbitrary material distribution shown in a. The element highlighted in $\mathbf{b}$ is subject to Top and Bottom Convection (TBC) across its lateral surfaces as well as Boundary Side Convection (BSC), as it is located along the domain boundary. The element highlighted in $\mathbf{c}$ is subject to TBC as well as Internal Side Convection (ISC) across its interface, $\Gamma_{i 1}$, with a neighboring void element.

\subsection{The optimization problem}

The mathematical formulation of the full topology optimization problem is stated as follows:

$$
\begin{aligned}
\underset{\rho}{\min .} & : b \\
& : \Phi_{0} \leq b \\
& : R_{s}^{\star} \cdot \Phi_{1} \leq b, \\
& : V(\boldsymbol{\rho}) / V_{0} \leq f \\
& : \mathbf{C} \boldsymbol{\theta}+\mathbf{K}_{\theta} \boldsymbol{\theta}=\mathbf{p}_{\theta}, \quad t \in\left[0 ; t^{*}\right] \\
& : \mathbf{K}(\boldsymbol{\theta}) \mathbf{u}=\mathbf{p} \\
& : 0 \leq \rho_{i} \leq 1, \quad i=1, \ldots, n
\end{aligned}
$$

where $\Phi_{0}$, is the undamaged (cold) compliance

$$
\Phi_{0}(\rho)=\phi\left(\rho, \mathbf{u}\left(\rho, \theta\left(\rho, t_{0}\right)\right)\right)
$$

and $\Phi_{1}$ is the hot compliance at time $t^{*}$

$$
\Phi_{1}\left(\rho, t^{*}\right)=\phi\left(\rho, \mathbf{u}\left(\rho, \theta\left(\rho, t^{*}\right)\right)\right)
$$

Here $\phi$ is the temperature dependent compliance

$$
\phi=\sum_{i=1}^{n}\left[E_{i}\left(\rho_{i}, \theta_{i}(\boldsymbol{\rho}, t)\right) \mathbf{u}_{e i}^{\top} \mathbf{K}_{e 0} \mathbf{u}_{e i}\right]
$$

where $\mathbf{u}_{e i}$ and $\mathbf{K}_{e 0}$ are the displacement vector of element $i$ and the stiffness matrix for an element with unit Young's modulus, respectively. Finally, we add a volume constraint given by:

$$
V=\sum_{i=1}^{n} v_{e} \rho_{i}
$$

where $v_{e}$ is the element volume (here equal for all elements) and $n$ is the number of design elements. 


\subsection{Adjoint sensitivity analysis}

The gradients of the objective function and the constraints are determined using adjoint sensitivity analysis. The problem is time dependent and gradient evaluation of the constraint with respect to design variables requires the solution of an adjoint system of equations backward in time. The analysis closely follows the one presented in [16, 38]. The main steps are presented below.

The sensitivities of $\Phi_{0}$ and $V$ are given as [5]:

$$
\begin{aligned}
\frac{\partial \Phi_{0}}{\partial \rho_{i}} & =-\mathbf{u}^{\top} \frac{\partial \mathbf{K}}{\partial \rho_{i}} \mathbf{u} \\
\frac{\partial V}{\partial \rho_{i}} & =v_{e}
\end{aligned}
$$

In order to determine the design sensitivity of $\Phi_{1}$, the temperature dependent compliance, $\phi$, is augmented by two sets of Langrangian multipliers, $\lambda_{u}$ and $\lambda_{\theta}$, multiplied by the residual of the static mechanical problem at time $t^{*}$ and the transient heat transfer problem:

$$
\begin{array}{r}
\Phi_{1}=\phi\left(\boldsymbol{\rho}, \mathbf{u}\left(\boldsymbol{\rho}, \boldsymbol{\theta}\left(\boldsymbol{\rho}, t^{*}\right)\right)\right)+\lambda_{u}^{\top}(\mathbf{p}-\mathbf{K u})+ \\
+\int_{0}^{t^{*}} \lambda_{\theta}^{\top}\left(\mathbf{p}_{\theta}-\mathbf{C}_{\theta} \dot{\boldsymbol{\theta}}-\mathbf{K}_{\theta} \boldsymbol{\theta}\right) \mathrm{d} t
\end{array}
$$

This holds for arbitrary $\lambda$, as the residuals are zero at equilibrium. Differentiating with respect to a design change, and using the chain rule, results in:

$$
\begin{aligned}
\frac{\mathrm{d} \Phi_{1}}{\mathrm{~d} \boldsymbol{\rho}_{i}}= & \mathbf{p}^{\top} \frac{\mathrm{d} \mathbf{u}}{\mathrm{d} \boldsymbol{\rho}_{i}}+ \\
& -\lambda_{u}^{\top} \mathbf{K} \frac{\mathrm{d} \mathbf{u}}{\mathrm{d} \boldsymbol{\rho}_{i}}-\lambda_{u}^{\top} \frac{\partial \mathbf{K}}{\partial \boldsymbol{\rho}_{i}} \mathbf{u}-\lambda_{u}^{\top} \frac{\partial \mathbf{K}}{\partial \boldsymbol{\theta}} \frac{\partial \boldsymbol{\theta}}{\partial \boldsymbol{\rho}_{i}} \mathbf{u} \\
& +\int_{0}^{t^{*}} \lambda_{\theta}^{\top}\left(\frac{\partial \mathbf{p}_{\theta}}{\partial \boldsymbol{\rho}_{i}}-\frac{\partial \mathbf{C}}{\partial \boldsymbol{\rho}_{i}} \dot{\boldsymbol{\theta}}-\frac{\partial \mathbf{K}_{\theta}}{\partial \boldsymbol{\rho}_{i}} \boldsymbol{\theta}\right) \mathrm{d} t \\
& +\int_{0}^{t^{*}} \lambda_{\theta}^{\top}\left(-\mathbf{C} \frac{\partial \dot{\boldsymbol{\theta}}}{\partial \boldsymbol{\rho}_{i}}-\mathbf{K} \frac{\partial \boldsymbol{\theta}}{\partial \boldsymbol{\rho}_{i}}\right) \mathrm{d} t
\end{aligned}
$$

Rearranging the terms and integrating line 4 of (24) by parts yields:

$$
\begin{aligned}
\frac{\mathrm{d} \Phi_{1}}{\mathrm{~d} \boldsymbol{\rho}_{i}}= & \left(\mathbf{p}^{\top}-\lambda_{u}^{\top} \mathbf{K}\right) \frac{\mathrm{d} \mathbf{u}}{\mathrm{d} \boldsymbol{\rho}_{i}}+ \\
& -\lambda_{u}^{\top} \frac{\partial \mathbf{K}}{\partial \boldsymbol{\rho}_{i}} \mathbf{u}-\lambda_{u}^{\top} \frac{\partial \mathbf{K}}{\partial \boldsymbol{\theta}} \frac{\partial \boldsymbol{\theta}}{\partial \boldsymbol{\rho}_{i}} \mathbf{u} \\
& +\int_{0}^{t^{*}} \lambda_{\theta}^{\top}\left(\frac{\partial \mathbf{p}_{\theta}}{\partial \boldsymbol{\rho}_{i}}-\frac{\partial \mathbf{C}}{\partial \boldsymbol{\rho}_{i}} \dot{\boldsymbol{\theta}}-\frac{\partial \mathbf{K}_{\theta}}{\partial \boldsymbol{\rho}_{i}} \boldsymbol{\theta}\right) \mathrm{d} t \\
& +\int_{0}^{t^{*}}\left(\lambda_{\theta}^{\top} \mathbf{C}-\lambda_{\theta}^{\top} \mathbf{K}\right) \frac{\partial \boldsymbol{\theta}}{\partial \boldsymbol{\rho}_{i}} \mathrm{~d} t-\left[\lambda_{\theta}^{\top} \mathbf{C} \frac{\partial \boldsymbol{\theta}}{\partial \boldsymbol{\rho}_{i}}\right]_{0}^{t^{*}}
\end{aligned}
$$

The first line of 25 is eliminated by requiring

$$
\mathbf{K} \boldsymbol{\lambda}_{u}=\mathbf{p}
$$

which results in $\lambda_{u}=\mathbf{u}$. The value of $\left[\lambda_{\theta}^{\top} \mathbf{C} \frac{\partial \theta}{\partial \boldsymbol{\rho}_{i}}\right]_{0}$ is zero as the initial temperature is not design dependent. The first term on the last line of (25) is eliminated by requiring

$$
\mathbf{C} \dot{\lambda}_{\theta}-\mathbf{K} \lambda_{\theta}=0, \quad \forall t \in\left[0, t^{*}\right]
$$


with terminal conditions obtained from the equality

$$
-\left.\lambda_{\theta}^{\top} \mathbf{C} \frac{\partial \boldsymbol{\theta}}{\partial \boldsymbol{\rho}_{i}}\right|_{t^{*}}-\left.\lambda_{u}^{\top} \frac{\partial \mathbf{K}}{\partial \boldsymbol{\theta}} \frac{\partial \boldsymbol{\theta}}{\partial \boldsymbol{\rho}_{i}} \mathbf{u}\right|_{t^{*}}=0
$$

The terminal value problem is transformed to an initial value problem by replacing $\lambda_{\theta}(t)$ with $\bar{\lambda}_{\theta}(\tau)$ where $\tau=t^{*}-t$

$$
\mathbf{C} \dot{\bar{\lambda}}_{\theta}+\mathbf{K} \bar{\lambda}_{\theta}=0, \quad \forall \tau \in\left[0, t^{*}\right]
$$

and the initial condition is:

$$
\bar{\lambda}_{\theta}(0)=\lambda_{\theta}\left(t^{*}\right)
$$

The derivative of $\mathbf{K}$ with respect to the temperature can be derived using (6). By satisfying the two adjoint problems (26) and (29), (25) reduces to:

$$
\begin{aligned}
\frac{\mathrm{d} \Phi_{1}}{\mathrm{~d} \boldsymbol{\rho}_{i}}= & -\lambda_{u}^{\top} \frac{\partial \mathbf{K}}{\partial \boldsymbol{\rho}_{i}} \mathbf{u} \\
& +\int_{0}^{t^{*}} \lambda_{\theta}^{\top}\left(\frac{\partial \mathbf{p}_{\theta}}{\partial \boldsymbol{\rho}_{i}}-\frac{\partial \mathbf{C}}{\partial \boldsymbol{\rho}_{i}} \dot{\boldsymbol{\theta}}-\frac{\partial \mathbf{K}_{\theta}}{\partial \boldsymbol{\rho}_{i}} \boldsymbol{\theta}\right) \mathrm{d} t
\end{aligned}
$$

The remaining derivatives with respect to the design variables are obtained using the interpolation expressions for the material properties, (4)-(6), and for the convection boundary terms (8)-(15).

\subsection{Filtering}

In order to regularize the solutions and ensure convergence to black and white designs, the design variables are filtered and projected to value 0 and 1 as introduced by Guest et al. [39]. The physical element densities are given by the projection [6]:

$$
\overline{\tilde{\rho}}_{e}=\frac{\tanh \beta \eta+\tanh \beta\left(\tilde{\rho}_{e}-\eta\right)}{\tanh \beta \eta+\tanh \beta(1-\eta)}
$$

where the projection sharpness is controlled by the parameter $\beta$ and the threshold value is determined by $\eta$. The filtered densities are found from:

$$
\tilde{\rho}_{e}=\frac{1}{\sum_{i \in N_{e}} H_{e i}} \sum_{i \in N_{e}} H_{e i} \rho_{i}
$$

where $N_{e}$ is the set of elements $i$ for which the center-to-center distance $\Delta(e, i)$ to element $e$ is smaller than the filter radius $r_{\min }$ and $H_{e i}$ is an inverse distance weight factor defined as:

$$
H_{e i}=\max \left(0, r_{\min }-\Delta(e, i)\right)
$$

The projection ensures that intermediate filtered densities smaller or larger than the threshold, $\eta$, are projected to 0 and 1, respectively. As reviewed in [6], $\eta=0$ imposes minimum length scale on the solid phase and $\eta=1$ imposes minimum length scale on the void phase. Selecting $\eta=0.5$ as in this paper ensures solid-void designs but neither guaranties solid nor void length scale. Hence, if a design later exhibits a certain length scale it is mainly due to the fire resistance constraint and not the filtering.

The physical densities, $\overline{\tilde{\rho}}$, are used to evaluate the objective function and the constraint functions, therefore the sensitivities with respect to the original design variables, $\rho$, are obtained using the chain rule as follows:

$$
\frac{\partial \psi}{\partial \rho_{j}}=\sum_{e \in N_{j}} \frac{\partial \psi}{\partial \tilde{\tilde{\rho}}_{e}} \frac{\partial \overline{\tilde{\rho}}_{e}}{\partial \tilde{\rho}_{e}} \frac{\partial \tilde{\rho}_{e}}{\partial \rho_{j}}
$$

where the sensitivity of the filtered density of element $e$ with respect to the design variables, is given by:

$$
\frac{\partial \tilde{\rho}_{e}}{\partial \rho_{j}}=\frac{1}{\sum_{i \in N_{e}} H_{e i}} H_{j e}
$$




\section{Results}

\subsection{A heat resistant $M B B$-beam problem}

This section extends the well-known minimum compliance Messerschmitt-Bölkow-Blohm (MBB)-beam problem, stated in Figure 7b in terms of a design domain and mechanical boundary conditions, with the thermal boundary conditions stated in Figure $7 \mathrm{a}$. Following the method presented in the previous section, a convection controlled heat flow is generated across the physical boundary, causing structural degradation. In order to prevent degradation of the mechanically loaded parts of the structure, the design domain boundary located near the mechanical load and support are thermally insulated. These parts of the structure may be considered fire protected.

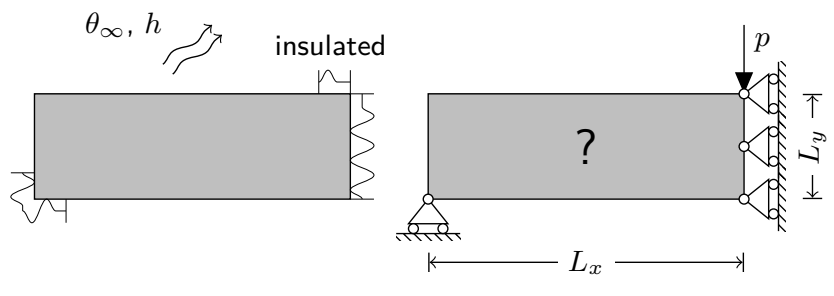

b

Figure 7: Heat resistant MBB-beam problem formulation in terms of thermal (a) and mechanical (b) boundary conditions, applied utilizing symmetry.

An extensive study for 32 increasing values of $t^{\star}$ is carried out with the following parameters: $L_{x}=5 \mathrm{~m}, L_{y}=$ $1.67 \mathrm{~m}, d=0.3 \mathrm{~m}, \theta_{\infty}=1000^{\circ} \mathrm{C}, h_{0}=10^{3} \mathrm{~W} / \mathrm{m}^{2} \mathrm{~K}, p=100 \mathrm{~N}, k_{0}=10 \mathrm{~W} / \mathrm{m} \mathrm{K}, c_{0}=10^{3} \mathrm{~J} / \mathrm{kg} \mathrm{K}, E_{0}=10^{5} \mathrm{~Pa}$, $\rho_{\text {mat }}=10^{4} \mathrm{~kg} / \mathrm{m}^{3}, \theta_{c r}=400^{\circ} \mathrm{C}, r_{\text {min }}=0.1 \mathrm{~m}, p_{k}=1, p_{c}=1, p_{E}=3, V^{\star} / v^{0}=0.5, R_{s}=1 / 5$. The structures are discretized by $n_{e l x}=300$ and $n_{e l y}=100$ bi-linear elements in the $x$ - and $y$-directions, respectively. In short, we optimize the MBB-beam for its fire resistance by requiring that the compliance after $t^{\star} \in[800,1475]$ s stays below 5 times the initial (cold) compliance. The artificial material properties are selected for demonstrating the methodology with relatively low computational resources. Realistic examples, subject to future research, require finer resolution and extensive utilization of high performance computing [40]. The structural response in the time domain is simulated using implicit Newmark time integration scheme $(\beta=0.5)$ with time step $t=25 \mathrm{~s}$ determined by convergence studies.

Pure minimum compliance design and 7 out of the considered 32 cases of fire resistant designs are shown in Figure 8 . Depending on case, using an even material distribution as starting guess sometimes results in sub-optimal topologies due to non-convexity of the optimization problem. In order to improve on these designs, the minimumcompliance reference structure, (a), is used as starting guess for optimization of structure (b) $\left(t^{\star}=800\right)$, structure (b) as starting guess for optimization of structure (c) $\left(t^{\star}=825\right)$, etc.. Initial (cold) compliances for the 32 structures are

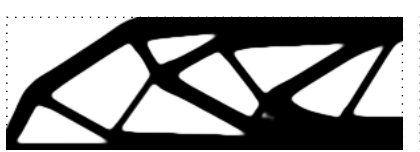

(a) $t^{\star}=-$

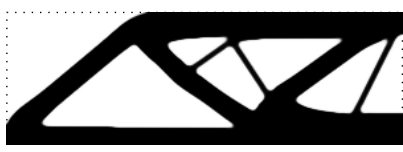

(z) $t^{\star}=1400 \mathrm{~s}$

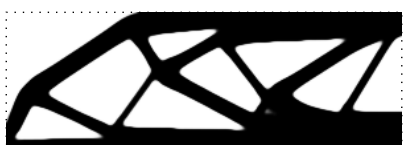

(b) $t^{\star}=800 \mathrm{~s}$

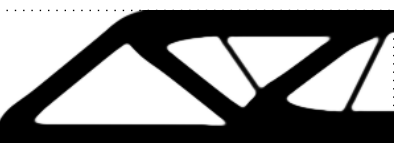

$(\alpha) t^{\star}=1425 s$

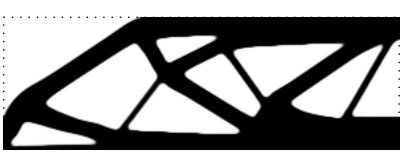

(r) $t^{\star}=1200 \mathrm{~s}$

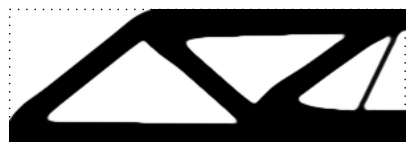

( $\beta) t^{\star}=1450 \mathrm{~s}$

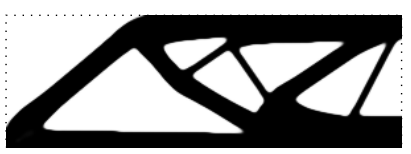

(v) $t^{\star}=1300 \mathrm{~s}$

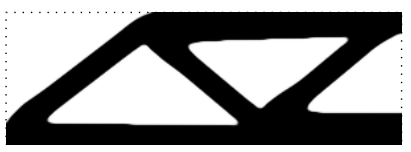

$(\gamma) t^{\star}=1475 s$

Figure 8: Structures, optimized with a varying evaluation time, $t^{\star}$. 


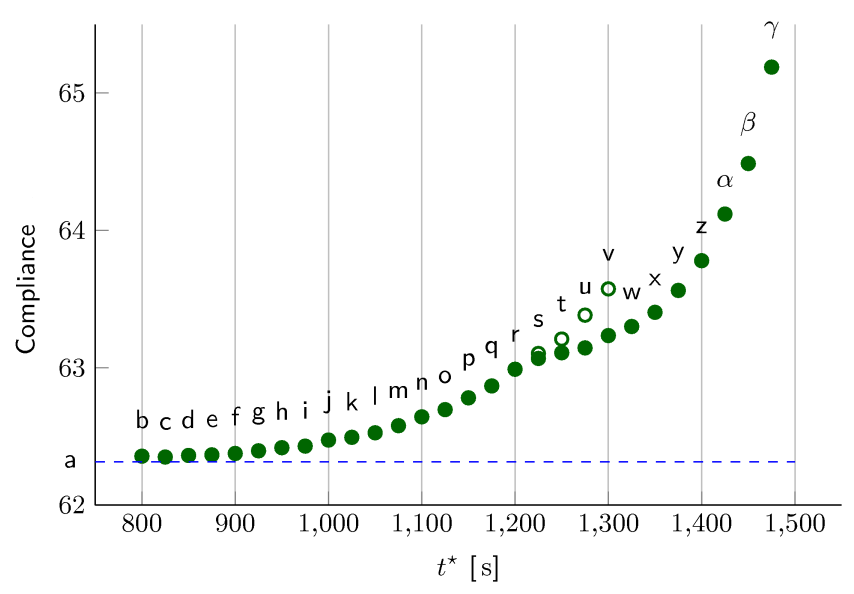

Figure 9: Cold compliance for the structures in Figure 8 The empty circles for designs (t), (u), and (v) represent sub-optimal designs obtained with initial guess design (r).

shown in Figure 9. It is interesting to observe that the resulting topologies are equal up to $t^{\star}=1200 \mathrm{~s}$ (point r). At this point, the small diagonal bar towards the left disappears and another, simpler design takes over up to $t^{\star}=1425$ (point $\alpha$ ). For $t^{\star}=1200 \mathrm{~s}$, starting from a uniform design makes the algorithm converge to a local minimum corresponding to the previous structures. However, using structure $\mathrm{w}$ as starting guess, one can recover better local minima with the simpler structures corresponding to points $\mathrm{v}, \mathrm{u}, \mathrm{t}$ and $\mathrm{s}$. Although, we do not know of similar systematic studies in the topology optimization literature, we are convinced that the observed behavior regarding convergence to local optima during topological transitions will hold for all kinds of topology optimization problems and is not limited to our proposed fire safety approach. By observing Figure 8, it is clear that increasing the requirement to fire survival time results in less structural detail since narrow bars will lose their stiffness faster than wide ones.

The compliance histories for the 8 structures from Figure 8 are shown in Figure 10. From an initial very low compliance of the cold structures, the curves increase in steps, corresponding to full degradation of individual elements of the structures, up to the gray band corresponding to 5 times the initial compliance.

The reference structure, (a), and the structure optimized for $t^{\star}=800 \mathrm{~s}$, (b), seem identical, but the compliance histories are quite different. Figure 11 illustrates the difference, where black and white indicate a positive and negative difference between (a) and (b), respectively, and gray means no difference. The main differences exist at the leftmost parts near the support. To further illustrate the differences, Figure 12 illustrates the amount of degraded material in the two structures (indicated with red), after 800s of heat loading. Here it is seen how the left-most horizontal bar and the inclined bar just above it are totally degraded for the reference design (a), whereas the inclined bar has survived the fire after $t^{*}=800 \mathrm{~s}$ for structure (b), explaining the differences in time-dependent compliance.

This example demonstrates how the proposed optimization strategy can control the survivability of a structure after a certain fire exposure. This ability comes at the cost of an increased compliance in the serviceability (cold) state. This increment, however, is very small and within the usual 5\% limit of design accuracy. On the other side, the fire resistance is more than doubled.

\subsection{A heat resistant Michell structure}

Next we design a "fire proof" Michell-structure with design domain and boundary settings stated in Figure 13 As before, a convection controlled heat flow is generated across the physical boundary, causing structural degradation.

Except for the following parameters, all values are the same as for the previous example: $L_{x}=5 \mathrm{~m}, L_{y}=4 \mathrm{~m}$, $n_{\text {elx }}=400, n_{\text {ely }}=320, r_{\text {min }}=0.0375 \mathrm{~m}, p_{k}=1, p_{c}=1, p_{E}=3, V^{\star} / V^{0}=0.3, R_{s}=\frac{1}{3}$, i.e., we optimize the Michell structure in order to maintain at least $1 / 3$ of the initial stiffness after time $t^{*}$.

As before we optimize a large number of structures using a continuation strategy for the starting guess, however, this time we only show the obtained structures in Figure 14 without a detailed study of compliance history and cold compliances. The figure shows how the initially aesthetic Michell structure with its fine and optimal grid work 


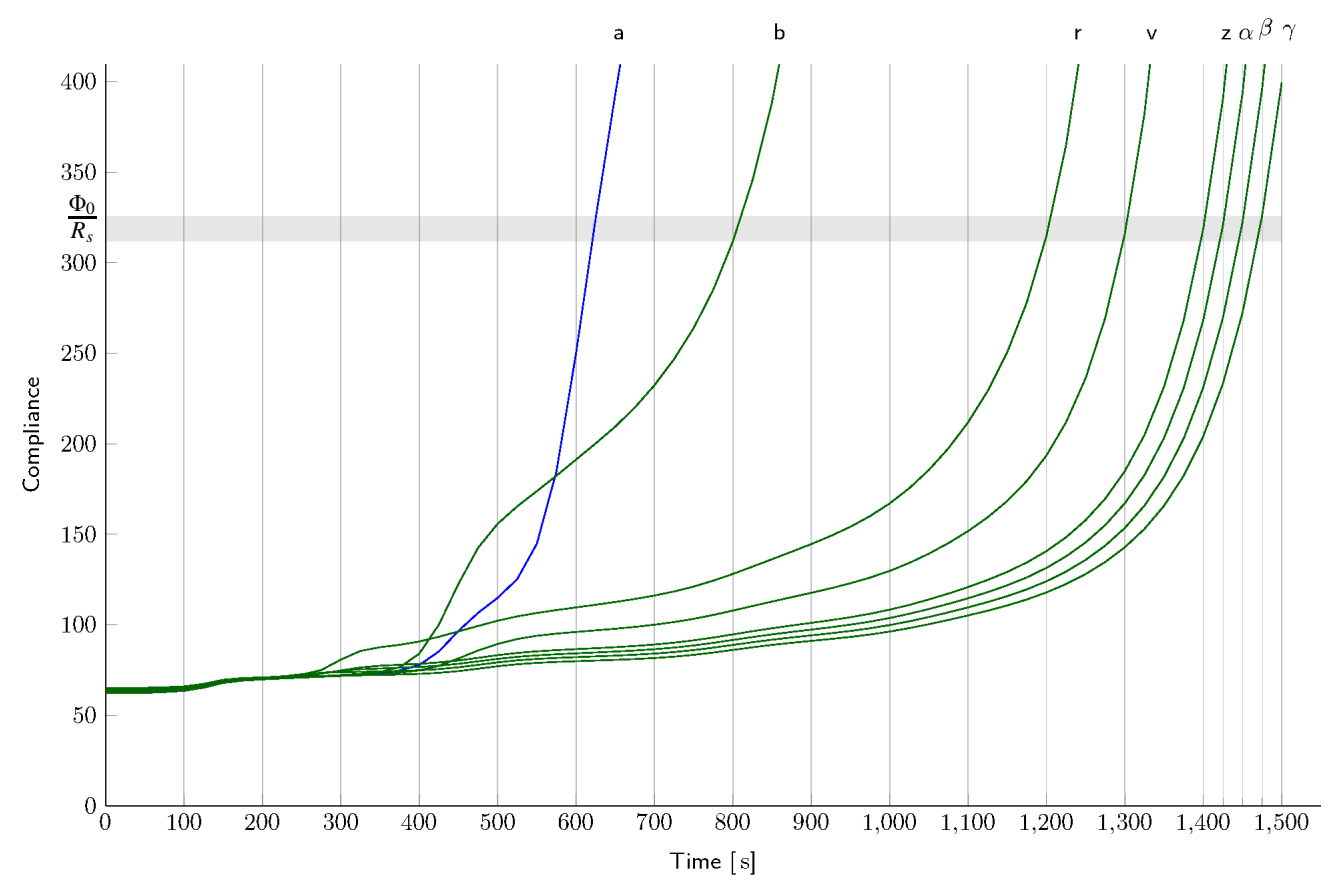

Figure 10: Compliance history for the structures in Figure 8

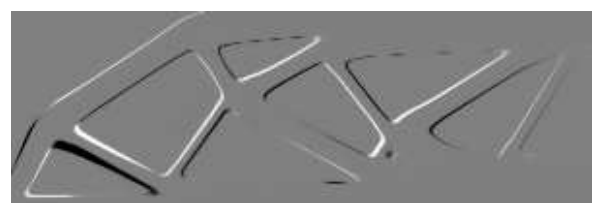

Figure 11: The difference in density between the reference structure, (a), and structure (b).

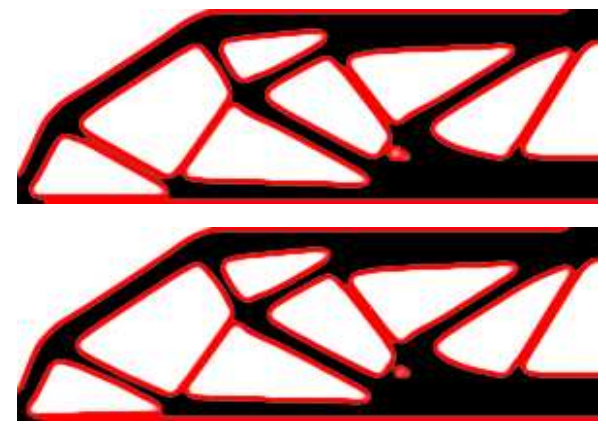

Figure 12: Reference structure, (a), and structure (b) after $800 \mathrm{~s}$ of heat loading. 


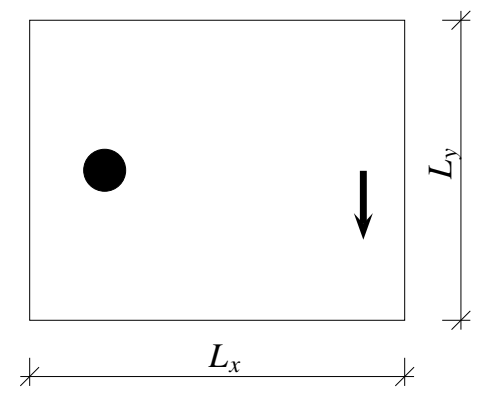

Figure 13: Heat resistant Michell-structure problem formulation. The structure is fixed in the black area.

degrades to a much simpler and crude looking structure which, however, will maintain its load carrying capability after the imposed exposure to fire. The cold compliance value for the initial structure (a) of 77.3 increases to 82.5 (i.e. less than $7 \%$ ) for the final structure $\zeta$, indicating that a constraint for fire safety not necessarily results in significant deterioration of the compliance at the serviceability load.

\subsection{A robust structure}

The final example focuses on the inclusion of a fire safety related criterion in the design of a larger-scale structure. Even if the example is still very academic, the analysis of a large compartment is of particular interest from the design point of view, given the inapplicability of current simplified design methods based on post-flashover fire models. The problem stated in Figure 15, in terms of a design domain, a passive solid domain, and thermal and mechanical boundary conditions, is used as an example. Convection is applied across all structural surfaces in the lower right quadrant of the design domain, simulating a local fire. This is considered a two-dimensional problem, e.g. a large domain depth with respect to the width and the height of the compartment is assumed and therefore convection is not applied on the lateral surfaces. Also, as this problem considers an internal fire in an enclosure, no convection is applied across the external design domain boundaries.

The reference structure shown in Figure 16 (a) is simply optimized for minimum (cold) compliance, whereas case (b) also complies with the fire safety constraint, $R_{s}=1 / 5$ at $t^{\star}=8400 \mathrm{~s}$. As $\Phi_{a 0}$ indicates in the graph, structure (a) performs best for the unheated condition. After approximately 1200 seconds of localized heat exposure structure (b) performs better. From here on, the compliance of (a) increases dramatically, as the lower right part of the structure can no longer carry the load. The compliance of (b), on the other hand, surpasses the allowed value of $\Phi_{0 b} / 0.20=3.929$ after exactly 8400 seconds. Evidently, the very thick central part of the structure together with the arch-like upper part provides the requested stiffness, despite most of the lower right part having failed due to the localized fire exposure.

The example shows how the method can be used to obtain robust structures by means of both thicker members and static redundancy. The method can easily be adapted to work with several fire scenarios instead of just one. This could be used to obtain structures that have a robust behavior with respect to multiple fire scenarios.

\section{Discussion and conclusions}

The applicability of topology optimization was extended to include structural fire safety design. The topology optimization method combined with a simplified planar fire model resulted in optimized fire resistant structures for a specified time period. As demonstrated, the optimized structures are simple and consist of few and thick bars in contrast to standard compliance designs that tend to have Michell-like fine grids. Therefore, the imposed fire model is similar to an erosion operator [36] and indirectly imposes a minimum length scale only on members preventing fire induced collapse of the structure, i.e., all elements ensuring the structural stability at the final stage before collapse possess minimum length scale. It is also interesting to note that the fire resistant design in this respect seems very different from the design against other accidental actions, such as explosions and impacts. These are dynamic and 


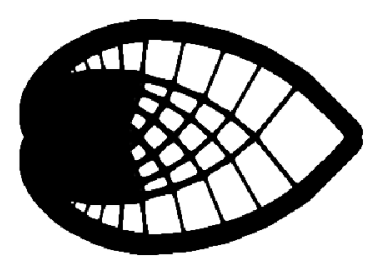

(a) $t^{\star}=-$

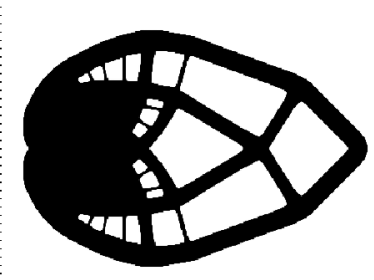

(r) $t^{\star}=1200 \mathrm{~s}$

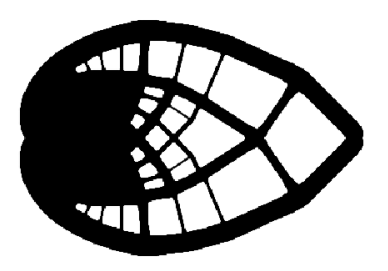

(b) $t^{\star}=800 \mathrm{~s}$

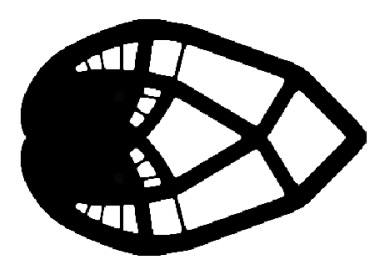

(v) $t^{\star}=1300 s$

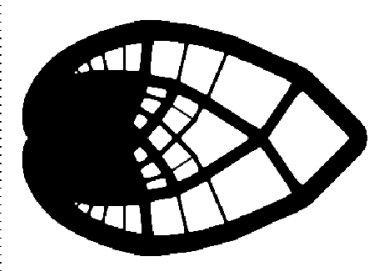

(j) $t^{\star}=1000 \mathrm{~s}$

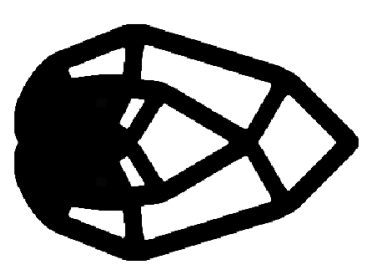

(z) $t^{\star}=1400 s$

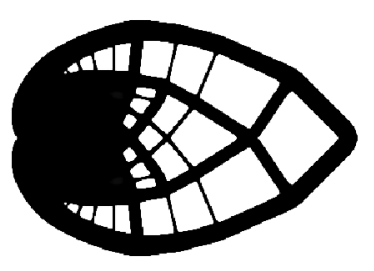

(n) $t^{\star}=1100 \mathrm{~s}$

Figure 14: Structures, optimized with a varying evaluation time, $t^{\star}$.

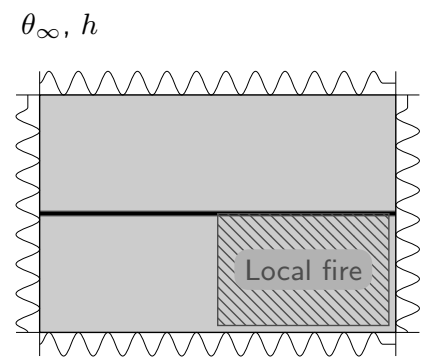

a

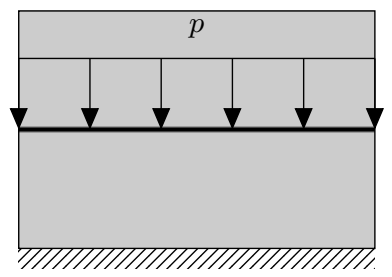

b

Figure 15: Problem formulation in terms of thermal (a) and mechanical (b) boundary conditions, approximating a local fire. A passive solid region is employed along the location of the line load. 
(a)
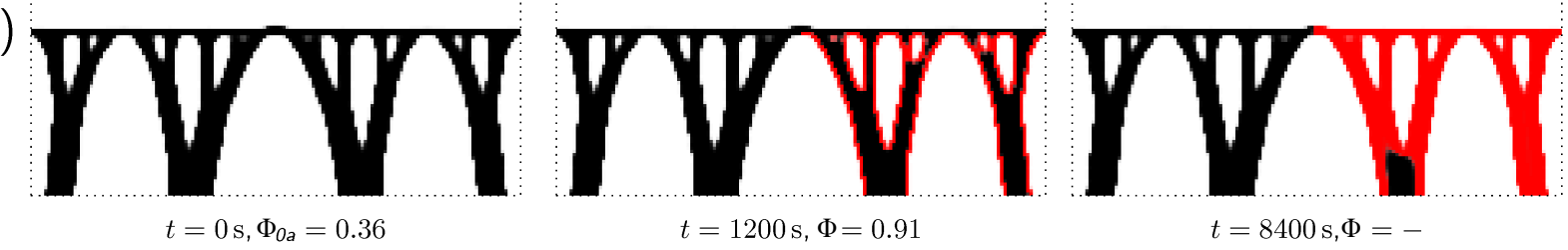

(b)

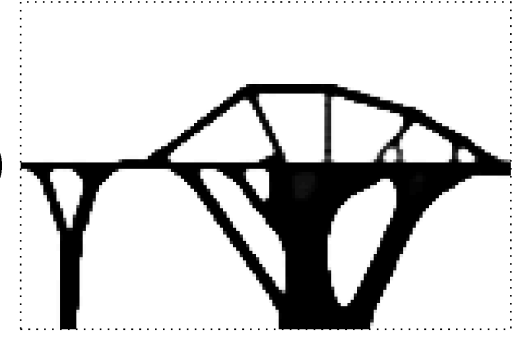

$t=0 \mathrm{~s}, \Phi_{0 b}=0.78$

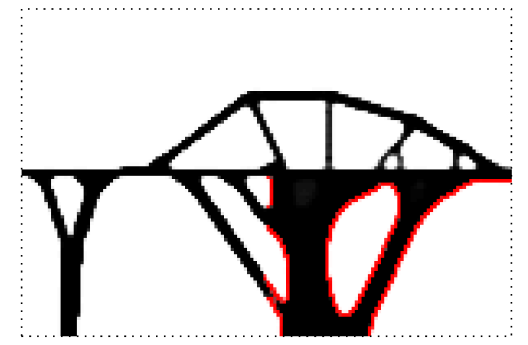

$t=1200 \mathrm{~s}, \Phi=0.97$

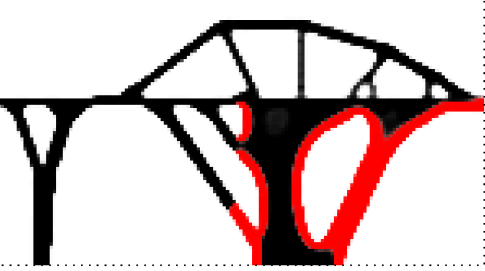

$t=8400 \mathrm{~s}, \Phi=3.92$

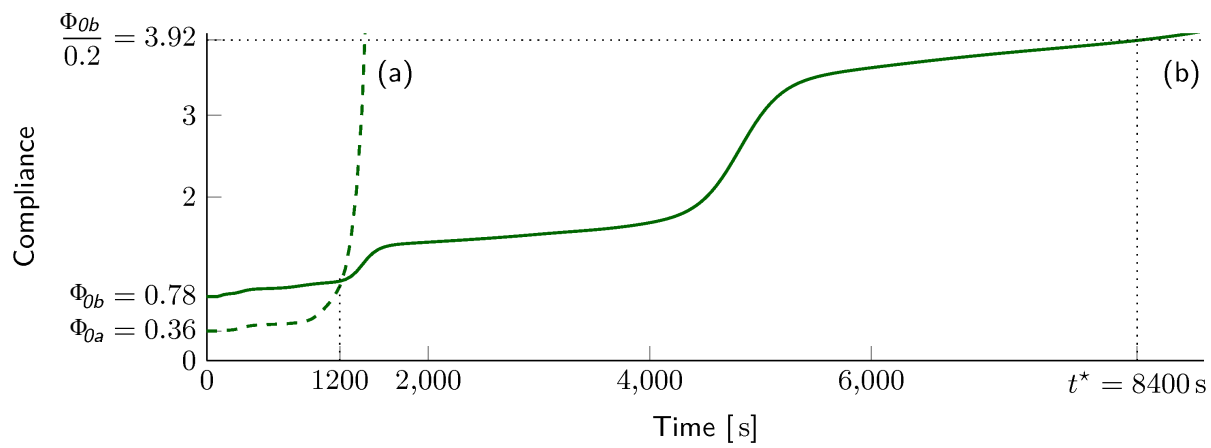

(b)

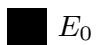

$E_{0}$

$E_{\min }$

$E_{\min }$

Figure 16: The graph shows the history of compliance for the two structures (a) and (b), that are capable of resisting a fire, localized in the lower right quadrant of the design domain, for different durations of time. The images show the temperature controlled degradation of stiffness, indicated by red. 
generally quite severe actions, but are often concentrated on one or a few neighboring elements. Thus, optimized structures for such types of actions tend to posses large number of thin redundant elements which ensure structural stability in case one or a few of them fail. Therefore, optimization of a full structure needs to take into account all possible load scenarios, degradation of the material properties, and changes of the structural layout during the accidental load states. Such optimizations will result in both safer and more economical design and are subject of future research.

\section{Acknowledgements}

The authors would like to thank the Villum Foundation for funding through the NextTop project and the TopTen project sponsored by the Danish Council for Independent Research (DFF-4005-00320). Valuable research and technical discussions with Joe Alexandersen are greatly appreciated.

\section{References}

[1] EN 1990, Eurocode 0 - Basis of structural design, Brussels: Comité Européen de Normalisation CEN, 2002.

[2] K. Hertz, Reinforcement data for fire safety design, Magazine of Concrete Research 56 (8) (2004) $453-459$.

[3] DS/EN 1991-1-2 DK NA, Danish National Annex to Eurocode 1: Part 1-2 Actions on structures exposed to fire, Erhvevs- og Byggestyrelsen, Denmark, 2011.

[4] M. P. Bendsøe, N. Kikuchi, Generating optimal topologies in structural design using a homogenization method, Computer Methods in Applied Mechanics and Engineering 71 (2) (1988) 197 - 224. doi:10.1016/0045-7825(88)90086-2

[5] M. P. Bendsøe, O. Sigmund, Topology optimization : theory, methods and applications, Springer, 2003.

[6] F. Wang, B. S. Lazarov, O. Sigmund, On projection methods, convergence and robust formulations in topology optimization, Structural and multidisciplinary optimization 43 (6) (2011) 767-784.

[7] B. S. Lazarov, F. Wang, O. Sigmund, Length scale and manufacturability in density-based topology optimization, Archive of Applied Mechanics 86 (1) (2016) 189-218. doi:10.1007/s00419-015-1106-4

[8] H. Rodrigues, P. Fernandes, Topology optimal design of thermoelastic structures using a homogenization method, Control and cybernetics 23 (1994) 553-563.

[9] O. Sigmund, S. Torquato, Composites with extremal thermal expansion coefficients, Applied Physics Letters 69 (21) (1996) $3203-3205$. doi:10.1063/1.117961

[10] J. Jonsmann, O. Sigmund, S. Bouwstra, Compliant thermal microactuators, Sensors and Actuators A: Physical 76 (13) (1999) 463 - 469. doi:10.1016/S0924-4247(99)00011-4

[11] L. Yin, G. Ananthasuresh, A novel topology design scheme for the multi-physics problems of electrothermally actuated compliant micromechanisms, Sensors and Actuators A: Physical $97 \quad-\quad 98$ (2002) $599 \quad-\quad 609$. doi:http://dx.doi.org/10.1016/S0924-4247(01)00853-6

[12] G. Ho Yoon, Y. Young Kim, The element connectivity parameterization formulation for the topology design optimization of multiphysics systems International Journal for Numerical Methods in Engineering 64 (12) (2005) 1649-1677. doi:10.1002/nme.1422 URL http://dx.doi.org/10.1002/nme.1422

[13] T. Bruns, Topology optimization of convection-dominated, steady-state heat transfer problems, International Journal of Heat and Mass Transfer 50 (2007) $2859-2873$.

[14] J. Alexandersen, Topology optimization for convection problems (2011).

[15] Y.-S. Chung, C. Cheon, S. Hahn, Reconstruction of dielectric cylinders using FDTD and topology optimization technique, Magnetics, IEEE Transactions on 36 (4) (2000) 956-959. doi:10.1109/20.877600

[16] J. Dahl, J. Jensen, O. Sigmund, Topology optimization for transient wave propagation problems in one dimension, Structural and Multidisciplinary Optimization 36 (6) (2008) 585-595. doi:10.1007/s00158-007-0192-5

[17] Y. Elesin, B. Lazarov, J. Jensen, O. Sigmund, Time domain topology optimization of 3D nanophotonic devices, Photonics and Nanostructures - Fundamentals and Applications 12 (1) (2014) 23 - 33. doi:10.1016/j.photonics . 2013.07.008

[18] S. Turteltaub, Optimal material properties for transient problems, Structural and Multidisciplinary Optimization 22 (2) (2001) 157-166. doi:10.1007/s001580100133

[19] C. B. Pedersen, Crashworthiness design of transient frame structures using topology optimization, Computer Methods in Applied Mechanics and Engineering 193 (68) (2004) 653-678. doi:10.1016/j.cma.2003.11.001

[20] A. Diaz, A. Benard, Topology optimization of heat-resistant structures, Vol. 2 A, 2003, pp. 633-639.

[21] O. Pettersson, S. Magnusson, J. Thor, Fire Engineering Design of Steel Structures, Lund Institute of Technology, Sweden, 1976.

[22] ISO834-1, Fire resistance tests - Elements of building construction - Part 1: General requirements for fire resistance testing, International Organization for Standardization (ISO), Geneva, Switzerland, 1999.

[23] EN 1991-1-2, Eurocode 1: Action on structures, Part 1-2: General actions - Actions on structures exposed to fire, Brussels: Comité Européen de Normalisation CEN, 2002.

[24] Energistyrelsen, Eksemplelsamling om brandsikring af byggeri (in Danish), Copenhagen: Klima-, Energi- og Bygningsministeriet, Energistyrelsen, 2012.

[25] F. Gentili, L. Giuliani, F. Bontempi, Structural response of steel high rise buildings to fire: System characteristics and failure, Journal of Structural Fire Engineering 4 (1) (2013) 9-26. 
[26] A. Usmani, Y. Chung, J. Torero, How did the WTC tower collapse: a new theory, Fire Safety Journal 38 721-744.

[27] L. Giuliani, I. Budny, Different design approaches to structural fire safety, Int. J. of Lifecycle Performance Engineering 1 (2) (2013) 135-158.

[28] B. Karlsson, J. Quintiere, Enclosure fire dynamics, CRC Press, 2000.

[29] K. Hertz, Parametric fires for structural design, Fire Technology 48 (4) 807-823.

[30] J. Stern-Gottfried, G. Rein, Travelling fires for structural design. Part I: Literature review, Fire Safety Journal 74 (1) $74-85$.

[31] DS-INF 146, Robustness - Background and principles - Information, Danish Standard Association, 2003.

[32] EN 1991-1-7, Eurocode 1, Part 1-7: General actions - Accidental actions, Brussels, Belgium: Comit Europen de Normalisation (CEN), 2006.

[33] ASCE/SEI 7-10, Minimum Design Loads for Buildings and Other Structures, Reston, VA, USA: ASCE Standard, Structural Engineering Institute of the American Society of Civil Engineers, 2010.

[34] U. Starossek, M. Haberland, Approaches to measures of structural robustness, Structure and infrastructure engineering 7 (7-8) (2011) 625631.

[35] R. D. Cook, D. S. Malkus, M. E. Plesha, R. J. Witt, Concepts and Applications of Finite Element Analysis, John Wiley \& Sons, 2001.

[36] O. Sigmund, Morphology-based black and white filters for topology optimization, Structural and multidiciplinary optimization 33 (4-5) (2007) 401-424. doi:10.1007/s00158-006-0087-x

[37] A. Verbart, M. Langelaar, F. van Keulen, A new approach for stress-based topology optimization: Internal stress penalization, in: $10^{t} h$ World Congress on Structural and Multidisciplinary Optimization, Orlando, Florida, USA, 2013.

[38] B. S. Lazarov, R. Matzen, Y. Elesin, Topology optimization of pulse shaping filters using the hilbert transform envelope extraction, Structural and Multidisciplinary Optimization 44 (3) (2011) 409-419. doi : 10.1007/s00158-011-0642-y

[39] J. K. Guest, J. H. Prvost, T. Belytschko, Achieving minimum length scale in topology optimization using nodal design variables and projection functions, International Journal for Numerical Methods in Engineering 61 (2) (2004) 238-254. doi:10.1002/nme.1064

[40] N. Aage, E. Andreassen, B. S. Lazarov, Topology optimization using PETSc: An easy-to-use, fully parallel, open source topology optimization framework, Structural and Multidisciplinary Optimization 51 (3) (2015) 565-572. doi:10.1007/s00158-014-1157-0 